Journal Club

Editor's Note: These short, critical reviews of recent papers in the Journal, written exclusively by graduate students or postdoctoral fellows, are intended to summarize the important findings of the paper and provide additional insight and commentary. For more information on the format and purpose of the Journal Club, please see http://www.jneurosci.org/misc/ifa_features.shtml.

\title{
Subthalamic Nucleus Cell-Specific Expression of Nicotinic Acetylcholine Receptors Uncovers Novel Basal Ganglia Microcircuits
}

\author{
Eric R. Starr, John F. Imbery, and Stuart A. Collins \\ Biomedical Sciences Program in Neurosciences and Neurological Disorders, Department of Neurosciences, University of Toledo College of Medicine and \\ Life Sciences, Toledo, Ohio 43614 \\ Review of Xiao et al.
}

The subthalamic nucleus (STN) is a part of the basal ganglia (BG) and thus has a role in modulating voluntary motor function. Dysfunction of STN neuronal activity is thought to underlie a variety of movement disorders. For example, in Parkinson's disease (PD), abnormalities in STN activity correlate with motor deficits like resting tremor and rigidity (Hamani et al., 2004). While surgical interventions aimed at physiologically restoring abnormal STN activity can work, these techniques are invasive and often ineffective (Wagle Shukla and Okun, 2014). Therefore, studies identifying potential pharmacological targets that can alter STN neuronal activity are exciting. In a recent article published in The Journal of Neuroscience, Xiao et al. (2015) identified a novel BG microcircuit based on expression of nicotinic acetylcholine receptors (nAChR) in distinct neuronal subpopulations within the STN. The findings demonstrate the potential role of STN neurons

\footnotetext{
Received May 1, 2015; revised June 15, 2015; accepted June 16, 2015

We thank Drs. Marthe Howard and Joseph Margiotta for their constructive criticism and guidance in the Journal Paper Review course in the Neurosciences and Neurological Disorders Graduate Program and for their advice in preparing this manuscript.

The authors declare no competing financial interests.

Correspondence should be addressed to Eric R. Starr, Department of Neurosciences, University of Toledo College of Medicine and Life Sciences, 3000 Arlington Avenue, Toledo, Ohio 43614. E-mail: Eric.Starr@rockets.utoledo.edu.

DOI:10.1523/JNEUROSCI.1707-15.2015

Copyright $\odot 2015$ the authors $\quad 0270-6474 / 15 / 3510645-02 \$ 15.00 / 0$
}

expressing $\alpha 4 \beta 2$-nAChRs in modulating neuronal activity in efferent targets.

Composed mainly of glutamatergic neurons, the STN primarily innervates the substantia nigra pars reticulata $(\mathrm{SNr})$ and the globus pallidus internus (GPi). Both the $\mathrm{SNr}$ and the GPi contain GABAergic efferent neurons projecting to thalamocortical neurons, which in turn facilitate activation of motor cortex neurons. When STN neurons fire, they increase activation of GPi and SNr neurons, leading to greater thalamocortical inhibition and ultimately inhibition of motor function (Hamani et al., 2004). The STN and the SNr also project to dopaminergic neurons in the substantia nigra pars compacta $(\mathrm{SNc})$, but the functional relevance of this circuit in motor control is unclear (Hamani et al., 2004).

Neurons in several of the aforementioned BG nuclei express $\alpha 4 \beta 2$ nAChRs, $\alpha 7 \mathrm{nAChRs,}$ and $\alpha 6 \beta 2$ nAChRs (Quik and Wonnacott, 2011). Chronic nicotine treatment increases expression of $\alpha 4 \beta 2 \mathrm{nAChRs}$ in the substantia nigra via posttranscriptional mechanisms (Marks et al., 1992). This upregulation has been attributed to increased $\alpha 4 \beta 2$-nAChR assembly and trafficking to the cell surface (Srinivasan et al., 2014). In conjunction with increasing $\alpha 4 \beta 2-\mathrm{nAChR}$ expression, chronic nicotine increases firing of $\mathrm{SNr}$ neurons, an effect that is correlated with increased suppression of SNc activity (Xiao et al., 2009). Because one of the primary afferents stimulating the $\mathrm{SNr}$ originate from neurons in the STN, Xiao et al.
(2015) sought to examine the effects of chronic nicotine exposure on STN neuronal function. In doing so, they identified novel subpopulations of nAChR-expressing neurons in the STN and mapped their functional connections to the $\mathrm{SNr}$ and the SNc.

Xiao et al. (2015) first examined nAChR expression in STN neurons by focally applying acetylcholine (ACh). They observed two distinct ACh-evoked responses in STN neurons. One subpopulation of neurons exhibited an ACh-evoked current characterized by fast activation and fast decay kinetics while another subpopulation exhibited an ACh-evoked current characterized by fast activation but slow decaykinetics. Treatment with the $\alpha 7-\mathrm{nAChR}$ antagonist, MLA, completely abolished AChevoked responses in neurons exhibiting a fast decaying current. Treatment with the $\beta 2$ nAChR antagonist $\mathrm{DH} \beta \mathrm{E}$ completely abolished ACh-evoked responses in neurons exhibiting a slow decaying current. From these experiments, it was concluded that $\alpha 7$ nAChRs and $\alpha 4 \beta 2$-nAChRs are expressed in distinct subpopulations of STN neurons.

Synaptic activity in the STN is modulated by inhibitory GABAergic inputs from the globus pallidus externus (GPe) as well as excitatory glutamatergic inputs from the motor cortex (Hamani et al., 2004). Therefore, Xiao et al. (2015) assessed the density of inhibitory and excitatory afferents innervating neurons expressing $\alpha 4 \beta 2$ - or $\alpha 7$-nAChRs. Analysis of spontaneous and evoked excitatory and inhib- 
itory postsynaptic events indicated that neurons expressing $\alpha 4 \beta 2-\mathrm{nAChRs}$ received a greater proportion of glutamatergic inputs whereas neurons expressing $\alpha 7$-nAChRs received a greater proportion of GABAergic input. Though not directly assessed, these findings suggest neurons expressing $\alpha 4 \beta 2$ $\mathrm{nAChRs}$ receive a larger proportion of inputs from the motor cortex while neurons expressing $\alpha 7$-nAChRs receive a greater proportion of inputs from the GPe. The differing inputs to the neuronal subpopulations suggest that STN neurons expressing $\alpha 4 \beta 2-n A C h R s$ or $\alpha 7$ $\mathrm{nAChRs}$ have different roles in BG motor circuitry.

The STN is part of the hyperdirect and indirect motor pathways of the BG, which are distinguished by the origin of inputs to the STN (Nambu et al., 2002). The hyperdirect pathway involves direct glutamatergic inputs from the motor cortex to the STN. These cortical afferents activate the STN, which, through its efferent targets to BG output nuclei, inhibit thalamocortical neurons regulating motor cortex output. The STN neurons expressing $\alpha 4 \beta 2$ nAChRs, which receive a larger proportion of glutamatergic innervations, may therefore be part of the hyperdirect pathway. STN neurons in the indirect pathway receive GABAergic inputs from the GPe. Activation of this pathway is thought to stop movements that have been activated (Nambu et al., 2002). The current findings, highlighting a stronger innervation of neurons expressing $\alpha 7$-nAChRs by GABAergic inputs, suggest that these cells may be more strongly associated with the indirect pathway. Further conclusions on the potential role of neurons expressing $\alpha 4 \beta 2$ - and $\alpha 7$-nAChRs in mediating voluntary movements would be strengthened by studies highlighting the origin and density of inputs to these neurons.

To further characterize the circuitry involving STN neurons expressing $\alpha 4 \beta 2$ or $\alpha 7$-nAChRs, Xiao et al. (2015) measured spontaneous EPSCs (sEPSC) in $\mathrm{SNr}$ and $\mathrm{SNc}$ neurons following activation of these nicotinic receptors. After demonstrating STN outputs to the SNr and SNc remained intact in slice preparations, the authors showed focal perfusion of an $\alpha 4 \beta 2$-nAChR agonist in the STN increased sEPSC frequency in SNr neurons without detectably changing sEPSC frequency in SNc neurons. In contrast, application of an $\alpha 7-n A C h R$ agonist in the STN resulted in a greater increase of sEPSC frequency in SNc than SNr neurons. The authors therefore proposed that STN neurons expressing $\alpha 4 \beta 2$-nAChRs preferentially project to the $\mathrm{SNr}$ while STN neurons expressing $\alpha 7$-nAChRs preferentially project to the SNc.

Previous findings by Xiao et al. (2009) showed that chronic nicotine treatment increased $\mathrm{SNr}$ firing and subsequently suppressed SNc activity. In light of this and their new discovery that STN neurons expressing $\alpha 4 \beta 2$-nAChRs innervate the SNr, Xiao et al. (2015) went on to examine the effects of chronic nicotine on $\alpha 4 \beta 2$ $\mathrm{nAChR}$ expression and the corresponding changes in neuronal activity in the STN. Chronic nicotine treatment increased $\alpha 4 \beta 2 \mathrm{nAChR}$ expression and increased the percentage of STN neurons expressing $\alpha 4 \beta 2$ nAChRs. Further analysis of STN neuronal activity both in vivo and in vitro showed increased firing of STN neurons expressing $\alpha 4 \beta 2$ - but not $\alpha 7$-nAChRs following chronic nicotine treatment. Thus, the current findings extend those of Xiao et al. (2009) by suggesting that the increase in activity of SNr neurons following chronic nicotine exposure may result from increased activity of STN neurons that express $\alpha 4 \beta 2$-nAChRs and synapse onto SNr cells. Though plausible, experiments are still needed to bolster this conclusion by determining whether increased activity in the $\mathrm{SNr}$ caused by chronic nicotine depends on changes in $\alpha 4 \beta 2$ nAChR function within STN neurons. Chronic nicotine also resulted in increased GABA release onto SNc dopaminergic neurons, presumably the result of increased activity of SNr neurons (Xiao et al., 2009). Because dopamine cell loss during $\mathrm{PD}$ is believed to occur, in part, through increased excitotoxicity (Caudle and Zhang, 2009), targeting $\alpha 4 \beta 2$ nAChRs may represent a useful therapeutic for reducing dopaminergic cell loss.

The chronic nicotine experiments performed by Xiao et al. (2015) suggest that targeting $\alpha 4 \beta 2$-nAChRs within the STN could be used to treat involuntary movement disorders by increasing STN activity. For instance, levodopa-induced dyskinesias during PD are thought to result from reduced activity in the STN (Hamani et al., 2004). This would result in decreased activity of downstream GABAergic neurons in the SNr and GPi, leading to a facilitation of thalamocortical motor pathways that may cause dyskinesias. Nicotine has been demonstrated to be effective in alleviating levodopa-induced dyskinesias in a PD model in monkeys, which could be a result of increased STN activity (Quik et al., 2007). Results from Xiao et al. (2009) and Xiao et al. (2015) support this hypothesis, in that chronic nicotine was demonstrated to concurrently enhance activity in the STN and its downstream target in the SNr. However, targeting $\alpha 4 \beta 2$-nAChRs may only work if there is a connection between STN neurons expressing $\alpha 4 \beta 2-n A C h R s$ and the GPi. Thus, future studies should investigate whether such a connection exists. Additionally, it should be determined whether $\alpha 4 \beta 2$ selective agonists mimic the effects of nicotine by upregulating $\alpha 4 \beta 2$-nAChRs within the STN. Using a selective agonist could reduce some off-target effects of nicotine.

The findings of Xiao et al. (2015) highlight a novel microcircuit based on differential expression of nAChRs on STN neurons. These findings suggest that targeting $\alpha 4 \beta 2$-nAChRs may have therapeutic implications for treating PD and other motor disorders.

\section{References}

Caudle WM, Zhang J (2009) Glutamate, excitotoxicity, and programmed cell death in Parkinson disease. Exp Neurol 220:230-233. CrossRef Medline

Hamani C, Saint-Cyr JA, Fraser J, Kaplitt M, Lozano AM (2004) The subthalamic nucleus in the context of movement disorders. Brain 127: 4-20. CrossRef Medline

Marks MJ, Pauly JR, Gross SD, Deneris ES, Hermans-Borgmeyer I, Heinemann SF, Collins AC (1992) Nicotine binding and nicotinic receptor subunit RNA after chronic nicotine treatment. J Neurosci 12:2765-2784. Medline

Nambu A, Tokuno H, Takada M (2002) Functional significance of the cortico-subthalamopallidal 'hyperdirect' pathway. Neurosci Res 43:111-117. CrossRef Medline

Quik M, Wonnacott S (2011) alpha6beta2* and alpha4beta2* nicotinic acetylcholine receptors as drug targets for Parkinson's disease. Pharmacol Rev 63:938-966. CrossRef Medline

Quik M, Cox H, Parameswaran N, O'Leary K, Langston JW, Di Monte D (2007) Nicotine reduces levodopa-induced dyskinesias in lesioned monkeys. Ann Neurol 62:588-596. CrossRef Medline

Srinivasan R, Henderson BJ, Lester HA, Richards CI (2014) Pharmacological chaperoning of nAChRs: a therapeutic target for Parkinson's disease. Pharmacol Res 83:20-29. CrossRef Medline

Wagle Shukla A, Okun MS (2014) Surgical treatment of Parkinson's disease: patients, targets, devices, and approaches. Neurotherapeutics 11:47-59. CrossRef Medline

Xiao C, Nashmi R, McKinney S, Cai H, McIntosh JM, Lester HA (2009) Chronic nicotine selectively enhances alpha4beta $2^{*}$ nicotinic acetylcholine receptors in the nigrostriatal dopamine pathway. J Neurosci 29:1242812439. CrossRef Medline

Xiao C, Miwa JM, Henderson BJ, Wang Y, Deshpande P, McKinney SL, Lester HA (2015) Nicotinic receptor subtype-selective circuit patterns in the subthalamic nucleus. J Neurosci 35:3734-3746. CrossRef Medline 